\title{
An Analytical-Numerical Approach to Model and Analyse Squirrel Cage Induction Motors
}

\author{
Alessandro Marfoli, Luca Papini, Paolo Bolognesi, Chris Gerada \\ Power Electronics, Machines and Control Group University of Nottingham \\ Nottingham, UK
}

\begin{abstract}
Nowadays the Finite Element Analysis (FEA) represents the most accurate tool available to investigate the electromagnetic operation of electric machines. However, when induced currents have to be calculated, such as in Squirrel-Cage Induction Machines (SCIM), a transient analysis may require a long elaboration time. Such issue is exacerbated when the machine features a skewed stator or rotor, since this requires at least a 2D multi-slice approximated analysis or even ultimately a full 3D model. In this paper, a general analytical method for modeling electromagnetic devices is applied to an industrial SCIM featuring a skewed rotor structure. The modeling approach is wisely implemented to pursue a fair balance between accuracy of the analysis and computational burden, taking advantage of all the symmetries existing in the structure of the machine to minimize the complexity of the model. The results provided by the model developed are compared with respect to the corresponding values provided both by FEA and by experimental tests carried out on the reference machine. Such comparison shows that the proposed model is actually able to achieve a pretty good balance between accuracy and computational efficiency.
\end{abstract}

Index Terms-Analytical model, Inductances, Induction Motor, Numeric resolution, Rotor cage, Slotting effects, Winding function

\section{INTRODUCTION}

Squirrel cage induction machines have been very popular for a long time in a wide range of sizes thanks to several interesting features such as manufacturing simplicity, relatively low cost, robustness, potential for direct line supply, possibility to operate at relatively high temperature, easy adaptation for high speed operation and fail-silent behavior when not fed [1], [2], [3]. In fact, SCIMs are still widely used for a lot of different applications, ranging from line-fed and inverterfed motors for industrial and civil applications to propulsion drives for transportation and even to power generation [**?]. Nevertheless, in many application fields more and more stringent requirements are emerging especially about efficiency of electrical machines: this is pushing the manufacturers to refine their designs to meet the expectations by better exploiting the mechanical and electromagnetic properties of the materials and by paying more attention to minimize the secondary phenomena that may affect unfavorably the performances, aiming to keep SCIMs competitive with respect to other solutions. In particular, the high frequency currents induced in the rotor cage due to the high order spatial harmonics exhibited by the stator windings represent a undesirable secondary effect which deteriorate the efficiency of the machine as they mainly flow in the upper portion of the solid bars due to skin and proximity effects, thus producing not-negligible additional
Joule losses. Moreover, the presence of high-order harmonics in the magnetomotive forces (MMFs) generated by the cage and stator windings produces asynchronous, synchronous and ripple torque components [4]. To reduce such parasitic effects mainly produced by the non-ideal structure of stator windings, SCIMs are often equipped with a skewed rotor, yet at the cost of a reduction of the mean torque produced. Therefore, models that are accurate enough to analyse the interactions between stator and rotor MMFs are required to quantify the impact on the machine performances and to permit adjusting its design aiming to mitigate the undesired secondary phenomena. The most accurate tool available for a detailed investigation of the SCIM operation is transient-with-motion FEA, but using systematically such tool is usually considered unpractical since investigating any specific steady-state condition may take a long computation time due to the necessity to await for the settling of the periodic currents induced in the rotor cage. When the machine is equipped with a skewed stator or rotor structure, an approximated multi-slice 2D-FEA or eventually a full 3D-FEA have to be used, further drastically increasing the computational burden, thus making even less attractive such solution. In fact, although accuracy is an important aspect, in practice any simulation model has to feature an adequate computational speed to turn out actually useful during any industrial design process.

On the other hand, the most commonly used model for SCIMs is the single-phase equivalent circuit (EC), which allows to predict the steady-state performance at different operating conditions under canonical symmetrical supply. However, the analytical computation of the lumped parameters included in the EC poses some issues due to complex secondary phenomena such as the non-linearity of magnetic materials and the uneven distribution of current across relatively large solid conductors such as the rotor bars. In fact, such phenomena vary with the operating conditions and cannot be neglected when the EC is going to be used to analyze a wide range of situations. The Time-Harmonic FEA (TH-FEA) has been demonstrated to be an effective tool to improve the characterization of the EC's parameters related to the fundamental spatial harmonic [5], [6], [7], [8], as it permits to obtain pretty accurate results with a limited computational effort. In literature are also reported multi-harmonic equivalent circuits where the lumped parameters are analytically computed per each harmonic order [9]. However, the calculation or separation of such parameters into their harmonic components by means of FEA has not yet been demonstrated to be an effective 
approach. Furthermore, representing the complex phenomena taking place inside SCIMs by means of a compact single-phase EC leads to a model that is inherently unable to provide much details about the actual internal condition of the machine. Alternatively, in [10] and [11], a mathematical derivation of the model for a squirrel cage winding in dq-reference frame is presented considering the distribution of bars. More recently, in [12] a modelling method for SCIMs based on stator and rotor windings functions has been developed and increased accuracy is achieved in [13] and [14], accounting for the slot openings effects by means of permeance function implemented as reported in [15].

According to the previous considerations on the modelling techniques for SCIMs, the development of a computationally efficient time-stepping simulation model able to predict the interactions between time and space harmonics, including slotting effects, as well as to analyze skewed structures, is deemed to be potentially very interesting. In particular, in this paper the general analytical model presented in [16] for the magnetic analysis of long-drum rotary electric machines is applied and tailored for the case of SCIMs to permit calculating the inductance matrix, developing further theoretical considerations with respect to [17] aiming to better keep into account the peculiar structure of the rotor cage winding and the effects of slotting and skewing. The efficient implementation of the proposed modeling approach is then addressed, focusing on achieving a suited balance between model accuracy and computational burden. A discussion of the model limitations is then presented, comparing significant results against corresponding data provided by different FEA models. Finally, simulation results obtained referring to an industrial induction machine are compared vs. experimental data obtained for the considered machine in the same conditions to assess the practical accuracy of the proposed modeling methodology.

\section{BACKGROUND}

A unified approach permitting to effectively model a generic electro-magneto-mechanical device by means of an equivalent circuit was presented in [16], highlighting the roles played by the different key functions purposely introduced. In particular, it was shown that the knowledge of the function giving the linked fluxes vs. currents and positions suffices to permit deriving a full electromagnetic and electromechanical model at device level. For the broad class of radial-flux machines, a general magnetic model permitting to approximately calculate the flux function above cited under suited hypotheses was presented in [18], highlighting again the roles played by the functions purposely introduced. A first combined application to SCIMs of the above general models was presented in [17] with promising results; therefore, a similar approach is adopted also in this paper, developing further considerations aimed to improve its implementation and to keep into account further features such as slot openings and skewing. The main aspects of the modeling approach adopted are briefly recalled in this section for the sake of clarity.

In general, it is assumed that the device to be analysed features a set of individual independent windings named "phases", each one similar to a simple curve whose terminals are close each other; any eventual connection among phases, e.g. Y-connection of armature, is treated separately, i.e. it is not embedded into the model. As shown again is Sec. III, also squirrel cages such as in SCIMs can be conceptually modeled this way. Such phases are labeled by sequential numbers and their corresponding relevant quantities (currents, linked fluxes, voltages) are piled-up into corresponding column vectors. The reference directions assumed for the quantities are according to load operation for voltages vs. currents and to right-hand rule for fluxes vs. currents. The phase currents and the Lagrangian variables determining the relative positions among the different parts of the device are assumed as state variables for the magnetic phenomena. In case of rotary machines, such as the examined SCIMs, the displacement angle $\alpha$ between stator and rotor can be selected as the Lagrangian variable. Therefore, the electrical equation relating the phase voltages $\bar{v}$ of the equivalent phases to their currents $\bar{i}$ can be expressed as

$$
\bar{v}=\mathbf{R} \bar{i}+\bar{M}(\alpha, \bar{i}) \frac{d \alpha}{d t}+\mathbf{L}(\alpha) \frac{d \bar{i}}{d t}
$$

where $\mathbf{R}$ is the resistance matrix and $\mathbf{L}(\alpha)$ is the inductance matrix, which depends only on the position $\alpha$ under the assumption of linear magnetic behaviour of the device. This may be achieved either exactly when all of the materials composing it feature such characteristic, or approximately when the m.m.f. drop inside the ferromagnetic materials composing the cores is negligible with respect to the m.m.f. drop across the layers of linear materials, in particular the airgap and eventually the magnets when present and operating within their reversible demagnetization area. The latter is usually the case, unless the device is operated determining heavy saturation at least in some significant parts: therefore, basically a linear magnetic operation will be assumed hereafter unless stated. The motional coefficient vector $\bar{M}(\alpha, \bar{i})$ in (1) is given by

$$
\bar{M}(\alpha, \bar{i})=\frac{\partial \mathbf{L}(\alpha)}{\partial \alpha} \bar{i}
$$

In general, the flux tubes found in the different parts of the device may be classified as main or secondary according to their relevance with respect to the electromechanical transformation, which is just related to the major or minor potential influence of positions on them as the generated electromechanical wrench is related to the variation of coenergy vs. positions. Therefore, a major role is played by the main flux tubes, which are those interconnecting the different bodies in the device crossing the gaps separating them. In an unskewed rotary machine, such flux tubes provide the principal part of the linked fluxes and thus of the inductance matrix, which was shown in [18] can be expressed as

$$
\mathbf{L}_{\mathbf{p}}(\alpha)=\ell \int_{0}^{1} \mu_{e}(\lambda, \alpha) \bar{N}_{e}(\lambda, \alpha) \bar{N}_{e}^{T}(\lambda, \alpha) d \lambda
$$

where $\lambda$ represents the normalized tangential position along the air gap mapping the span $[0,1), \ell$ is the axial length of magnetic cores of the machine, $\mu_{e}(\lambda, \alpha)$ is the equivalent permeability function and $\bar{N}_{e}(\lambda, \alpha)$ is the winding function vector. 
The equivalent permeability function accounts for the thickness along the main field lines, for the tangential width along the mean surface and for the passive magnetic properties of the layer/s interposed between the stator and rotor cores, thus highlighting any magnetic anisotropy eventually arising: when no magnets are present, such as in SCIMs, it is given by

$$
\mu_{e}(\lambda, \alpha)=\mu_{0} \cdot \frac{\tau_{g}(\lambda, \alpha)}{\epsilon_{g}(\lambda, \alpha)}
$$

where $\epsilon_{g}(\lambda, \alpha)$ represents the length of the portions of main flux lines connecting the stator and rotor magnetic cores, while $\tau_{g}(\lambda, \alpha)$ describes the tangential extension of the mean surface in the airgap layer, which is approximately assumed to be magnetically equipotential and such that the local value of magnetic field equals its average value along the considered portion of main field line. Both functions may depend on the tangential coordinate $\lambda$ and eventually on the position $\alpha$.

Each component of the vector winding function $\bar{N}(\lambda, \alpha)$ describes the distribution of its active sides along the tangential direction, thus featuring a step-wise dependence on $\lambda$. The equivalent winding function $\bar{N}_{e}(\lambda, \alpha)$, which actually counts in (3), is obtained by subtracting the weighted average as follows:

$$
\bar{N}_{e}(\lambda, \alpha)=\bar{N}(\lambda, \alpha)-\int_{0}^{1} \frac{\mu_{e}(\lambda, \alpha)}{\int_{0}^{1} \mu_{e}(\lambda, \alpha) d \lambda} \bar{N}(\lambda, \alpha) d \lambda
$$

In general, the electromagnetic wrench can be obtained as the partial derivative of the coenergy $C_{E}(\alpha, \bar{i})$ with respect to the Lagrangian position, turning into a simple second order polynomial expression for magnetically linear devices. In the considered case, the torque results equal to:

$$
W_{E}(\alpha, \bar{i})=\frac{\partial C_{E}(\alpha, \bar{i})}{\partial \alpha}=\frac{1}{2} \bar{i}^{T} \bar{M}(\alpha, \bar{i})
$$

Considering that in induction machines the windings can be grouped into stator and rotor sets, they can be numbered sequentially, thus permitting to split the vectors and matrix quantities introduced as follows:

$$
\begin{gathered}
\bar{i}(t)=\left[\begin{array}{c}
\bar{i}_{s}(t) \\
\bar{i}_{r}(t)
\end{array}\right], \quad \bar{\psi}(t)=\left[\begin{array}{c}
\bar{\psi}_{s}(t) \\
\bar{\psi}_{r}(t
\end{array}\right], \quad \bar{v}(t)=\left[\begin{array}{c}
\bar{v}_{s}(t) \\
\bar{v}_{r}(t)
\end{array}\right], \\
\mathbf{L}(\alpha)=\left[\begin{array}{ll}
\mathbf{L}_{s s}(\alpha) & \mathbf{L}_{s r}(\alpha) \\
\mathbf{L}_{r s}(\alpha) & \mathbf{L}_{r r}(\alpha)
\end{array}\right], \quad \mathbf{R}=\left[\begin{array}{cc}
\mathbf{R}_{s s} & \mathbf{0} \\
\mathbf{0} & \mathbf{R}_{r r}
\end{array}\right]
\end{gathered}
$$

\section{ROTOR CAGE MODEL}

The basic structure of a complete squirrel cage winding consists in a number $b$ of parallel conductors (bars) connected at both ends through shared low resistance paths (rings). Therefore, while the stator phases are composed of simple conductive paths whose terminals are clearly identified, at a first view the rotor conductive paths are not easily recognised as proper phases due to their articulated structure. Therefore, the modeling of the rotor cage has to be properly analyzed first. To such purpose, a useful topological representation can be obtained by sketching the cage in planar form as shown in Fig. 1, where the solid rotor bars and rings are ideally replaced by thin paths for the induced currents: these are shaped as radial segments representing the bars, which connect the internal and external circles that represent the rings. Such ideal representation of the cage is intended as an approximation which hides the peculiar phenomena characterising solid conductors, such as the non-uniform spatial distribution of current density across the section and the partial linking of leakage fluxes. The $3 b$ branch currents in the above graph are constrained by the first Kirchoff law: therefore, it is convenient to express them via a set of biunivocally correlated unconstrained currents. In particular, considering the planar nature of the graph, as shown in Fig. 1 the inner ring mesh and the $b$ external meshes delimited by adjacent bars represent the natural meshes of the graph: according to circuits theory, the associated set of $b+1$ loop currents constitutes then a suited and convenient set of unconstrained currents. Moreover, considering that each mesh is a simple closed curve, from an electromagnetic point of view the squirrel cage can be represented as a set of $b+1$ equivalent phases that are shortcircuited and inside which the corresponding loop currents above cited will flow. Therefore, it is worth noting that the variety of behaviours that a squirrel cage can potentially exhibit exceeds by far that of a 3-phase winding, since usually the number of bars included in a squirrel cage is far superior than 2. However, usually the mesh current associated to the inner ring may be considered negligible, except under very particular circumstances: therefore, the equivalent phases to be considered for representing the cage winding will be typically reduced to the $b$ external loops.

\section{A. Resistance Matrix}

According to the adopted simplified representation of the squirrel cage, the equivalent phase related to each external loop share parts of its path, i.e. the 2 bars, with the 2 adjacent phases. In particular, when the bars and bar loops are labelled with consecutive numbers as shown in Fig. 1, bar $k$ will be shared between the two adjacent meshes $k, k-1$, meaning that the related resistive voltage will depend on both of such mesh currents. Therefore, the resistance sub-matrix $\mathbf{R}_{R}$ related to the rotor equivalent phases features the following tri-diagonal structure

$$
\mathbf{R}_{R}=2 p\left[\begin{array}{cccc}
R_{d} & R_{m}^{\prime} & & R_{m}^{\prime} \\
R_{m}^{\prime} & R_{d} & R_{m}^{\prime} & \\
& \ddots & \ddots & \ddots \\
R_{m}^{\prime} & & R_{m}^{\prime} & R_{d}
\end{array}\right]
$$

a)
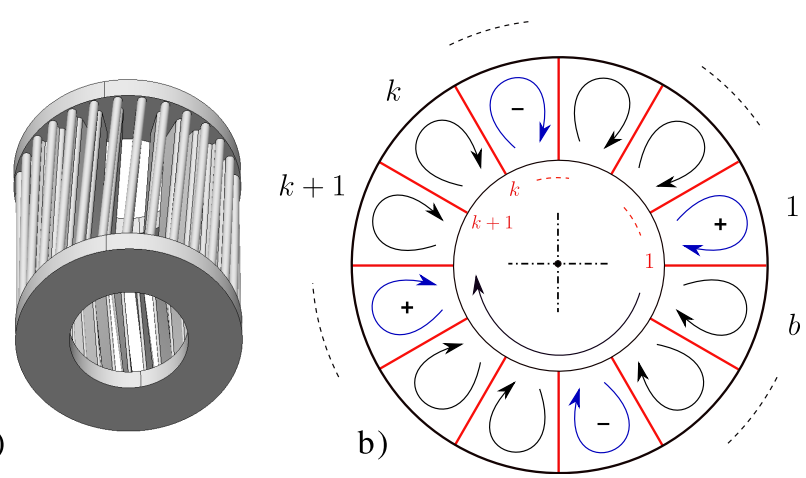

Fig. 1: a) Rotor cage structure and b) its planar representation. 
where $R_{m}^{\prime}=-R_{m}, R_{d}=2\left(R_{m}+R_{R i n g}\right), R_{m}$ is the resistance of each bar and $R_{\text {ring }}$ is the resistance of each ring sector.

In reality, the value of the above resistances would be actually affected by the operating conditions due to the bulk nature of the cage bars and rings. Nevertheless, the bars are located within the slots whereas the rings are only adjacent to the rotor core by one side. This means that the rings are affected by non-uniform current distribution much less than the bars: therefore, as a first approximation the value of the resistance of each ring sector can be simply estimated as

$$
R_{\text {ring }}=\rho \frac{\pi D_{\text {ring }}}{b S}
$$

where $\rho$ is the electrical resistivity while $D_{\text {ring }}$ and $S$ are diameter and cross section of the ring, respectively.

\section{B. Inductance Matrix}

Interesting considerations about the inductance matrix components related to the rotor structure (8) can be drawn applying the Gauss law in its integral form to a closed surface generated by joining the elementary surfaces single out by the $b$ bar loops (Main portion), which give rise to a quasi-cylindrical surface, and the two corresponding quasi-circular plain surfaces delimited by the rings (Caps portions):

$$
\nabla \cdot \hat{B}=0 \rightarrow \iint_{\text {Main }} \hat{B} \cdot \hat{n} d S+\iint_{\text {Caps }} \hat{B} \cdot \hat{n} d S=0
$$

In fact, when corresponding the flux crossing the Main surface can be expressed as in (12):

$$
\iint_{\text {Main }} \hat{B} \cdot \hat{n} d S=\sum_{k} \psi_{r k}=\overline{1}^{T} \bar{\psi}_{r}
$$

where $\overline{1}$ is a vector whose elements are all equal to 1 . As a first approximation, apparently the fluxes crossing the Caps portions might be neglected. Nevertheless, considering (11) and (12) such assumption would imply $\overline{1}^{T} \bar{\psi}_{r}=0$ meaning that the fluxes linked to the considered equivalent rotor phases turn out to be not linearly independent. Therefore one should conclude that in this way the matrix $\mathbf{L}_{\mathbf{p}}(\alpha)$ would turn out to be singular for any $\bar{i}$ and $\alpha$, since

$$
\overline{1}^{T}\left[\begin{array}{ll}
\mathbf{L}_{r s}(\alpha) & \mathbf{L}_{r r}(\alpha)
\end{array}\right]\left[\begin{array}{c}
\bar{i}_{s} \\
\bar{i}_{r}
\end{array}\right]=0 \quad \forall \quad(\bar{i}, \alpha)
$$

Such modelling approach would make impossible to analyse the dynamic behaviour of rotor equivalent phase currents when only stator supply is imposed, as usual. Therefore, the full circuital representation of an electrical machine equipped with a cage, must account for the inductive effects of end connections (leakage inductances) in order to make the model useful in most of cases. As a second level of approximation, the related leakage fluxes can be considered as mainly due to the self-inductance effect of the currents flowing in each ring sector delimited by adjacent bars, i.e. the mutual inductance effect may be neglected. The self-inductance of each ring sector may be estimated in different ways; for the scope of this paper, it was determined through FE analysis [19], obtaining the value $L_{\text {ring }}=2.7 \cdot 10^{-7}[\mathrm{H}]$ which was then taken into account within the matrix $\mathbf{L}_{r r}(\alpha)$.

\section{Rotor skewing}

The stator or rotor skewing is an effective method to reduce high order harmonics induced in both cage and stator windings. The ferromagnetic structure is axially twisted along the motor axis without changing the shapes of the cross section. For this reason, the computation of the performance of skewed motor by means of FEA is an heavy task from a computational point of view thus requiring 3D-model or alternative 2D-model featuring an axial discretisation of the problem in multi-slices. The latter is proved to be effective and less time consuming than full 3D problem, but it will be affected by not negligible errors unless an high number of slices is chosen. Since the skewing rate is usually small, the magnetic conditions at each cross section of the machine along the axial direction will not be much different from the 2D scenario considered in the analytical model recalled in Section II: therefore, a quasi-2D field map is expected to depends on the axial position, whereas the currents will be obviously the same in all of the sections of each bar or stator phase. Therefore, the general results recalled in Section II may be adapted to approximately analyse also a skewed machine by making all the descriptive functions depending on a linear coordinate spanning along the machine axis in the interval: $z \in[0, l]$. This leads to modify (3) into the general expression (14) for the inductances related to the main flux tubes:

$$
\hat{\mathbf{L}}(\alpha)=\int_{0}^{\ell} \int_{0}^{1} \mu_{e}^{\prime}(\lambda, z, \alpha) \bar{N}_{E}^{\prime}(\lambda, z, \alpha) \bar{N}_{E}^{\prime T}(\lambda, z, \alpha) d \lambda d z
$$

The above expression can be approximated in discrete form by considering a finite number of slices $n_{S}$ each one intended to represent the average contribution of an axial portion of the machine having length equal to $l_{i}$. Assuming that only the rotor is skewed, as usual in squirrel cage machines, the global inductance can be estimated according to (15)

$$
\hat{\mathbf{L}}(\alpha)=\sum_{i=1}^{n_{S}} \frac{\ell_{i}}{\ell} \mathbf{L}\left(\alpha-\theta_{s k, i}\right)
$$

where $\theta_{s k, i}$ is the skew angle of rotor in the direction of the coordinate $\alpha$ at the axial position of slice $i$ and $\ell_{i}$ is the length of the rotor portion attributed to such slice. In this paper such approach was used assuming review

$$
\begin{gathered}
\theta_{s k, i}=\frac{i-1}{n_{S}-1} \theta_{s k} \quad i=1 \ldots n_{s} \\
l_{i}=\frac{l}{n_{S}-1} \quad i=2 . . n_{S}-1 \quad l_{1}=l_{n_{S}}=\frac{l}{2\left(n_{S}-1\right)}
\end{gathered}
$$

where $\theta_{s k}$ is the total skew angle and the slices are assumed to be equally spaced with the first and the last one located respectively at the beginning and end of the machine active length. Thanks to the lower computational burden involved in the use of the analytical model with respect to the 2D FE analysis, the application of such approach permits using a high number of slices aiming to improve the results accuracy. 


\section{Model reduction in case of symmetries}

The stator usually features a symmetrical structure according to the number of pole pairs $p$. Moreover, often the squirrel cage features a fully symmetrical structure with identical bars evenly spaced and connected by regularly shape rings. When it happens that $b=p k$ with $k \in \mathbb{N}$, i.e. there is an integer number for each pole pair, one should expect that the currents flowing in the cage also feature a symmetrical pattern according to the pole pairs symmetry. Furthermore, sometimes it happens that the number of bars per pole pair is even: in such case, one should expect that the currents flowing in any pair of bars displaced by one pole pitch have just opposite waveforms. In such condition, the equivalent circuital modelling of the cage can be simplified by enforcing the above constrains among currents: This may be achieved by connecting in series all of the equivalent loop phases located 2 pole pitches apart, and eventually in anti-series all of the loop phases displaced by 1 pole pitch. In fact, as shown in Fig. 1, the current flowing in loops located at a pole pitch (i.e. the one indicated in blue) will be crossed by the same current with opposite sign while the current flowing in loops located at a pole pair pitch will be equal. This property thus permits to virtually connect in series and anti-series loops with the same current module of amplitude as shown in Fig. 2.

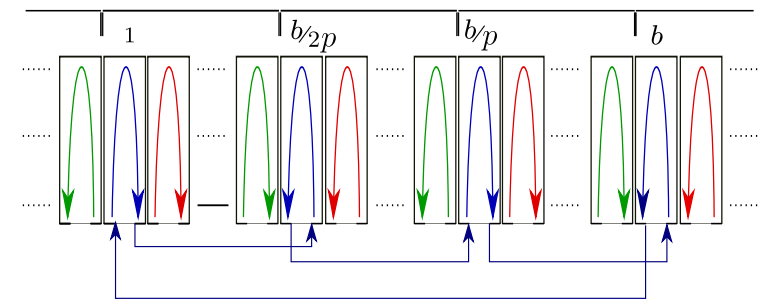

Fig. 2: Virtual loops connections of the same equivalent phase

The number of variables can be therefore drastically reduced from $b$ to $b /(2 p)$, thus simplifying the model complexity and the computational effort required. It is worth to note that, as already mentioned in [20], the new version of rotor winding functions shown in Fig. 3 permits to nullify the whole set of mutual-inductances between rotor phases leading to a rotor matrix inductances which assumes a diagonal structure. Such simplification in addition to reduce the model complexity, allows the matrix $\mathbf{L}_{r r}(\alpha)$ to be not singular due to the fact that only the main diagonal hosts elements different by zero. Therefore, the calculation of the ring inductance necessary to invert the matrix $\mathbf{L}(\alpha)$ in case of a full cage model as reported in Section III-B is deceived when a simplified model exploiting symmetries and anti-symmetries is adopted yet allowing a straightforward comparison against a 2D-FE simulation.

\section{NUMERICAL IMPLEMENTATION}

The analytical model is implemented numerically in MatlabSimulink platform. The stator and rotor windings representation and the modelling of the slotting effects necessary to accurately predict the performances of the machine are reported in this Section IV-A and IV-B. The enhancements made with respect to [18] are kept generic to be suitable for any combination of stator and rotor slots number and different angles of skewing.

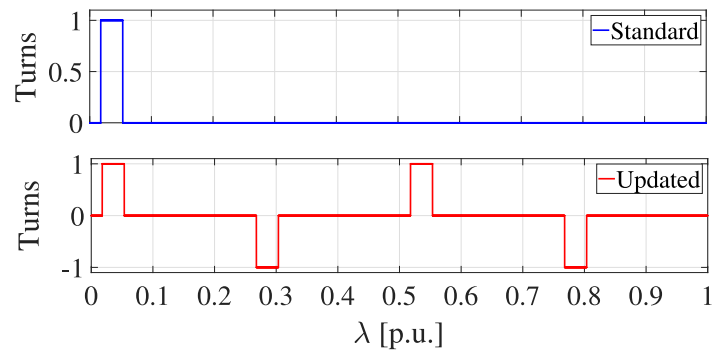

Fig. 3: Rotor WF exploiting the symmetries

\section{A. The winding functions}

An accurate implementation of the winding functions (5) permits to represent the multi harmonic nature of the MMFs. In [18], the circuital modelling technique is restricted to electrical machines featuring phase windings hosted in slots with small openings. In case of stator or rotor layouts with not negligible slot openings dimension, the conductors can be considered uniformly distributed along the opening width as represented in Fig. 4 where, the profile of the obtained updated WF plotted in red differs from the ideal case standard WF, draw with blue line. This modification directly impact on the harmonics components introduced in the model improving the results accuracy.

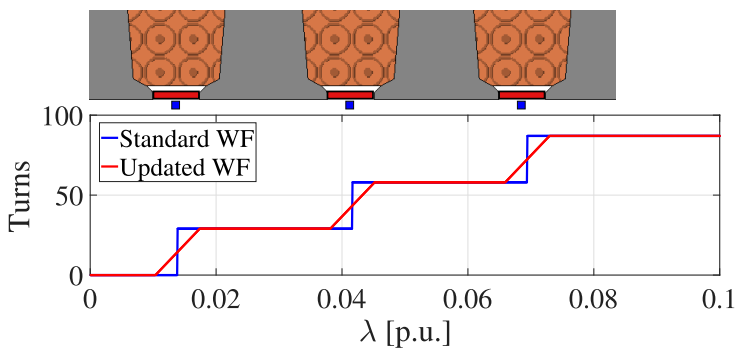

Fig. 4: Stator WF: standard vs updated version

\section{B. Slotting effects}

The modelling of the slot openings is also accounted in the computation of the inductances by means of the equivalent permeability function $\mu_{e}(\alpha, \lambda)$ reported in (4). Considering the small anisotropy introduced by the slot openings and air gap radius far greater than the slot openings depths might suggest the assumption of a cylindrical magnetic equipotential surface $\tau_{g}(\alpha, \lambda)$. However, such curve must lay orthogonal to the flux lines within the air gap $\forall \lambda$ as shown in the graphical example of Fig. 6a). To achieve a good representation the expressions (18) is implemented where, $r_{s}(\lambda, \alpha)$ and $r_{r}(\lambda, \alpha)$ are the equivalent inner stator and outer rotor radii.

$$
\begin{gathered}
\tau_{g}(\lambda, \alpha)=2 \pi \cdot \frac{r_{s}(\lambda, \alpha)+r_{r}(\lambda, \alpha)}{2} \\
\epsilon_{g}(\lambda, \alpha)=r_{s}(\lambda, \alpha)-r_{r}(\lambda, \alpha)
\end{gathered}
$$

As shown in the graphical representation of Fig. 6b), the length of the flux lines into the air gap change according to $\lambda$ and $\alpha$ coordinates. Such quantity is considered and implemented by means of the function $\epsilon_{g}(\lambda, \alpha)$, calculated as in (19) where, $r_{s}(\lambda, \alpha)$ and $r_{r}(\lambda, \alpha)$ are represented graphically in Fig. $\left.6 \mathrm{~b}\right)$. 


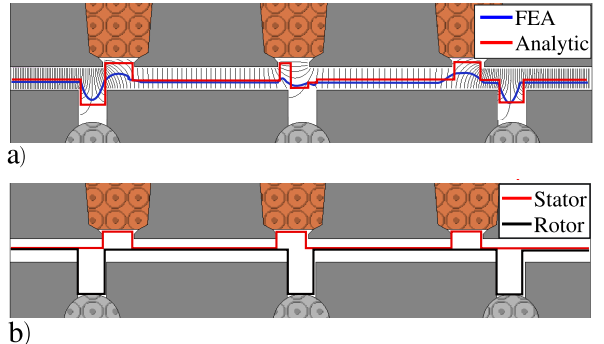

Fig. 6: a) Comparison between FE flux lines and idealised equipotential surface $\tau_{g}$ and b) graphical representation of $\varepsilon_{s}$ and $\varepsilon_{r}$ functions.

\section{Model Resolution}

The induced nature of the rotor currents requires a numerical resolution of the analytical model. Therefore, the electromagnetic system of equations reported in (1), is implemented in Matlab-Simulink platform where the inductances are calculated off-line and are organized in a look-up table. The input variables are the time evolution of the Lagrangian variable $\alpha(t)$ and the vector of the imposed voltages $\bar{v}(t)$; the vector of the currents are the outputs.

\section{Model Validation And Limitations}

The analytical-numerical modelling approach proposed in this paper is validated for a medium size 3-phase, $50[\mathrm{~Hz}]$, SCIM with a rated power of $11[\mathrm{~kW}]$, featuring 2 pole pairs, distributed single layer stator windings and equipped with a rotor cage consisting of a set of 28 short circuited rotor bars skewed by $\theta_{s k}$ electrical degrees. A peculiarity of the motor under investigation is that the bars are hosted in closed slots as shown in Fig. 7. However, to be consistent with the model assumptions [18], the rotor bridges are considered opened. This is justified by the fact that the electromagnetic saturation of the bridges makes the relative permeability of the material to drastically drop. The tuning of the analytical model is required selecting the slot opening width in order to match the leakage inductance value of the close bridges configuration at rated operating condition. Deviations in the predicted results in term of stator current waveform and steady state torque, respect to FE results and test are highlighted and commented in this section.

The analytical results are firstly compared against the FEA for $0<s<1$ with $s \in \mathbb{R}$. To highlight potentialities and limits of the proposed model, the predicted performance analytically computed are compared against four different FE models each one featuring different peculiarities. In TABLE I the characteristics featuring the FE models are summarized. Open (A-B-C) and closed (D) rotor bridges are considered in order to assess the impact of the slot openings functions. The model featuring idealised conductor (A) is compared with the solid bar ones (B-C-D) in order to account for secondary phenomena due to the skin effect. The impact of the material non-linearities (C-D) is assessed with respect to the model featuring magnetically linear material behaviour (AB). The analytical model developed in the previous section results close to Model A which is used for validation. The discrepancies that arise comparing the numerical-analytical solutions against the models $\mathrm{B}, \mathrm{C}$, and D is important to quantify the impact of the effects which are not included in the analytical model. As final validation test results of the motor with skewed rotor cage are compared against the analytical model.

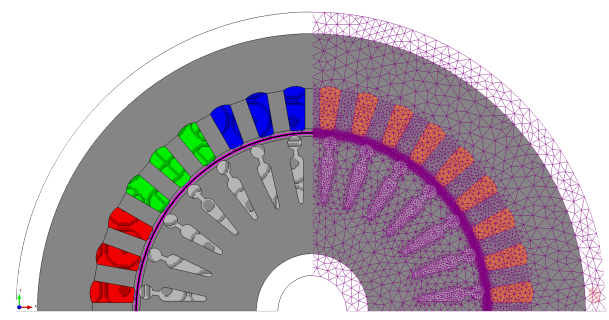

Fig. 7: A pole pair representation of the SCIM investigated
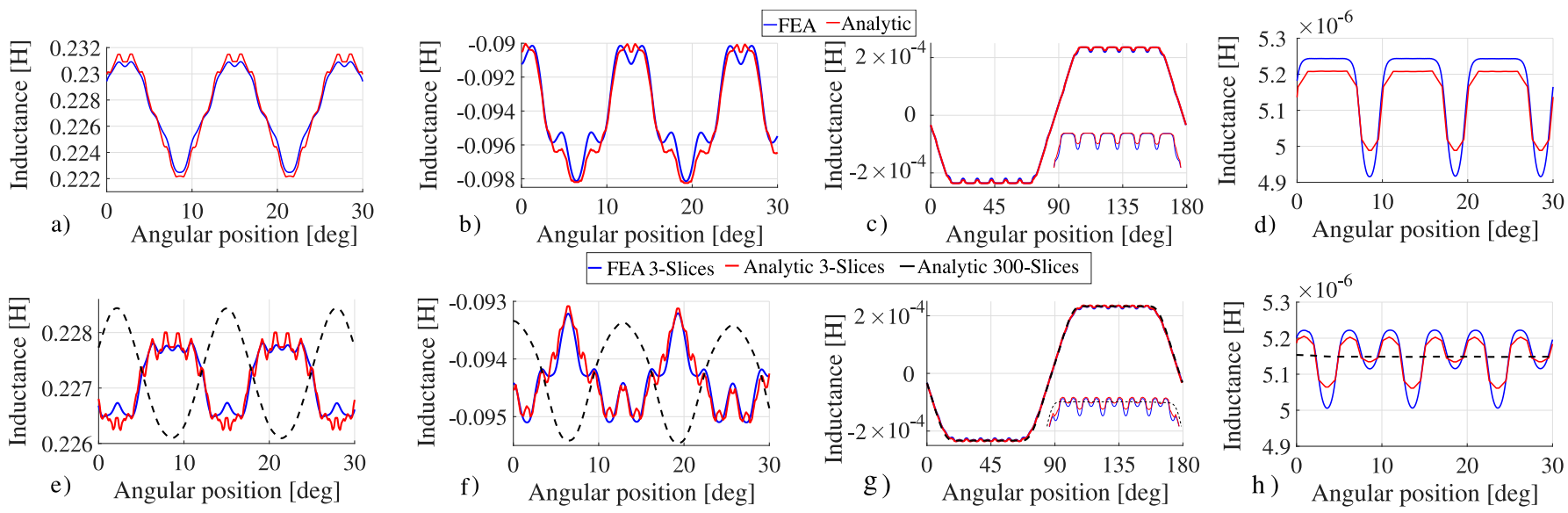

Fig. 5: Inductances vs angular position: a) Self stator inductance, b) mutual stator inductance, c) mutual stator-rotor inductance, d) self rotor inductance, e) self stator inductance skewed, f) mutual stator inductance skewed, g) mutual stator-rotor inductance skewed, h) self rotor inductance skewed. 


\section{A. Inductances Identification}

The inductances variation with respect to the rotor position $\alpha$ computed analytically are validated in the following. The Model A is considered for the comparison and solved as 2D Model in the time-stepping domain. Fig. 5 presents the results where a fairly good match is highlighted for the sample inductances components selected. The rotor skewing impacts over the whole set of inductances therefore, considering a constant skewing angle of $\theta_{s k}=\tau_{p}=1 / 36$, a time-stepping multi-slice FEA, modelling the machine with 3 slices is performed. It is important noticed that the number of slices selected to represent the skewing might affect the harmonics components of the inductances. For this reason, the analytical expression (15) is implemented for $n_{S}=3$ to match the slice number of the FE model. A farily good match is exhibited in Fig. 5 where $\theta_{s k, i}=-\theta_{s k} / 2$ for $i=1$. An high number $n_{S}$ is selected in the analytical computation in order to closely replicate the integral form (14). The results are shown with black line and compared in Fig. 5.

TABLE I: FE models

\begin{tabular}{l|ccc} 
& Bridges & Simulation & Bars \\
\hline Model A & Open & Linear & Stranded \\
Model B & Open & Linear & Solid \\
Model C & Open & No-linear & Solid \\
Model D & Close & No-linear & Solid
\end{tabular}

\section{B. FE Validation}

The analytical-numerical model is solved on a Intel Xeon CPU with clock frequency of $3.5 \mathrm{GHz}$ and $128 \mathrm{~GB}$ of RAM and the results are compared against the correspondent FE models, namely Model A and Model B. The stator current waveforms and the steady state torque are the benchmark quantities considered for the validation. A very accurate discretisation of a complete rotational period is obtained by dividing the coordinate $\lambda$ in 25200 divisions. Due to the simplified cage model introduced, the machine description is based on a state variable vector $\bar{i}$ whose dimension can be shrunk from 31 to 10 . Stator current waveforms are compared for $s=0.02$ and $s=0.04$ and reported in Fig. 8. The current waveforms predicted by the analytical model show an excellent match with respect to the idealized Model A with stranded bars in both the operating conditions, thus confirming the good representation of the inductances reported in Fig.5.

The steady state electromagnetic torque obtained numerically by applying (6) at different slip values is reported against the FE computation of Model A and Model B, in Fig. 9. The solid rotor bars featuring the cage of Model B affect the output torque, mainly due to the rotor resistance matrix which becomes a frequency-dependant quantity. A correction factor $\kappa_{e c}\left(f_{e, r}\right)$ for the rotor resistance (9) is therefore introduced in order to account for the skin effect. This factor is computed by means of specific simulations as described in [7].

The good agreement of the curves reported in Fig. 9, highlights how the proposed model allows to account for the stator and rotor harmonics interaction producing torque components

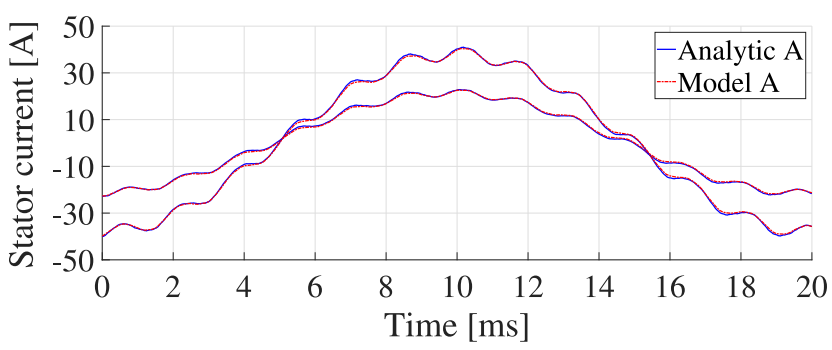

Fig. 8: Stator current waveforms at $s=0.02$ and $s=0.04$.

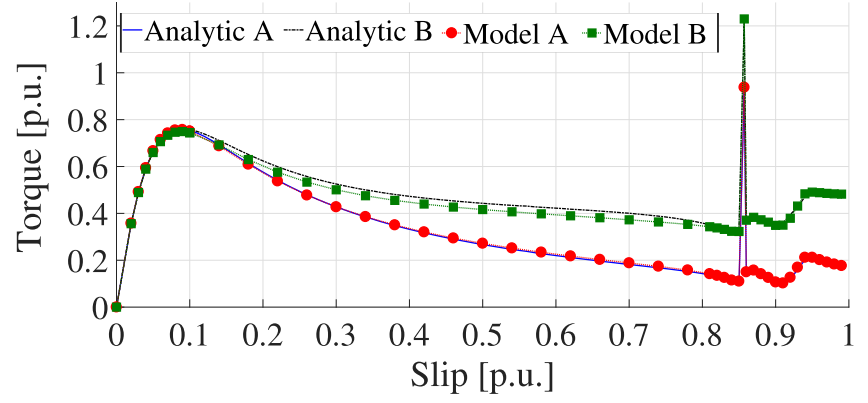

Fig. 9: Output torque against $s$.

together with the skin effects of the rotor bars for the operating points considered, yet remarkably reducing the computational burden with respect to the FE approach.

\section{Model Limitations}

The limitations of the analytical method are empathised when comparing it with respect to models featuring non-linear magnetization curves. The harmonic spectrum deviation with respect to the Model C in Fig. 11 is limited to few harmonic orders as the magnetic cores of the SCIM are dimensioned to operate below the saturation knee point. The discrepancy is however enhanced considering the effect of closed rotor slots in combination with the non-linear material behaviour as in Model D. The severe saturation that occurs in the bridges is highlighted in Fig. 10 and reflects into the peaks of specific harmonics (i.e. $6 h \pm 1$ with $h \in \mathbb{N}$ ) of the stator current.

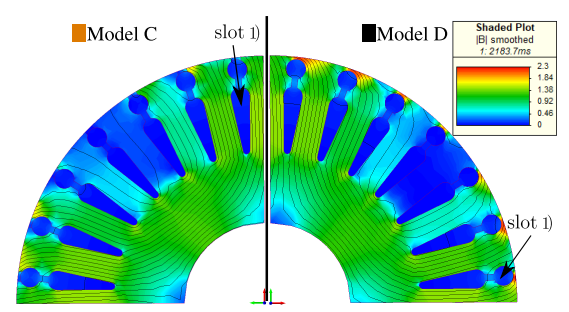

Fig. 10: Comparison of rotor field maps at $s=0.02$.

The saturation of the bridges plays an important role in the dependency of the leakage flux with respect to the current, in the containment of the current magnitude during the starting condition, in the reduction of the magnetizing current and high frequency losses at no-load, and consequently in the efficiency and power factor improvements achieved [21]. Moreover, the bridge saturations change location and magnitude with respect to the rotor position, slip value and magnetic load, thus leading to a dissimilar behaviour of the cage with respect to the models with opened slots. However, the model tuned at the 
rated operative point guarantees good agreement of SCIM's output at low slip values as shown in Fig. 12, when small deviations of the bridge saturation is contained with respect to the reference case at nominal slip. The analytical steady state torque in Fig. 12 exhibits a discrepancy for slip values higher than $s=0.1$ due to the non-linearity of the soft magnetic materials. However, the model is capable to represent fairly the trends of the asynchronous and synchronous torques due to the high order harmonics interactions at low rotor speeds.

\section{Skewed Model and Experimental Validation}

The experimental platform consists of a DC machine acting in driving mode directly coupled with the SCIM as shown in Fig. 13. Stator current waveforms are measured at different mechanical power values. The stator current waveforms are compared against the analytical results. The SCIM under test is equipped with a skewed rotor structure and therefore, both analytical and FE models are updated to account for skewing considering $n_{S}=300$ and $n_{S}=5$ slices, respectively.

In Fig. 14 the analytical stator current spectra at three different mechanical operative points are compared against multi-slice FE model and experimental test results. Although the analytical model proposed is based on the assumption of linear behaviour of the iron materials, the overall harmonic amplitudes show a fairly good match. However, the harmonic amplitudes discrepancy for $6 h \pm 1$ harmonic orders, related with the rotor bridges saturation, confirm again the limit of the analytical approach in predicting particular harmonics as mentioned previously in Section V-B.
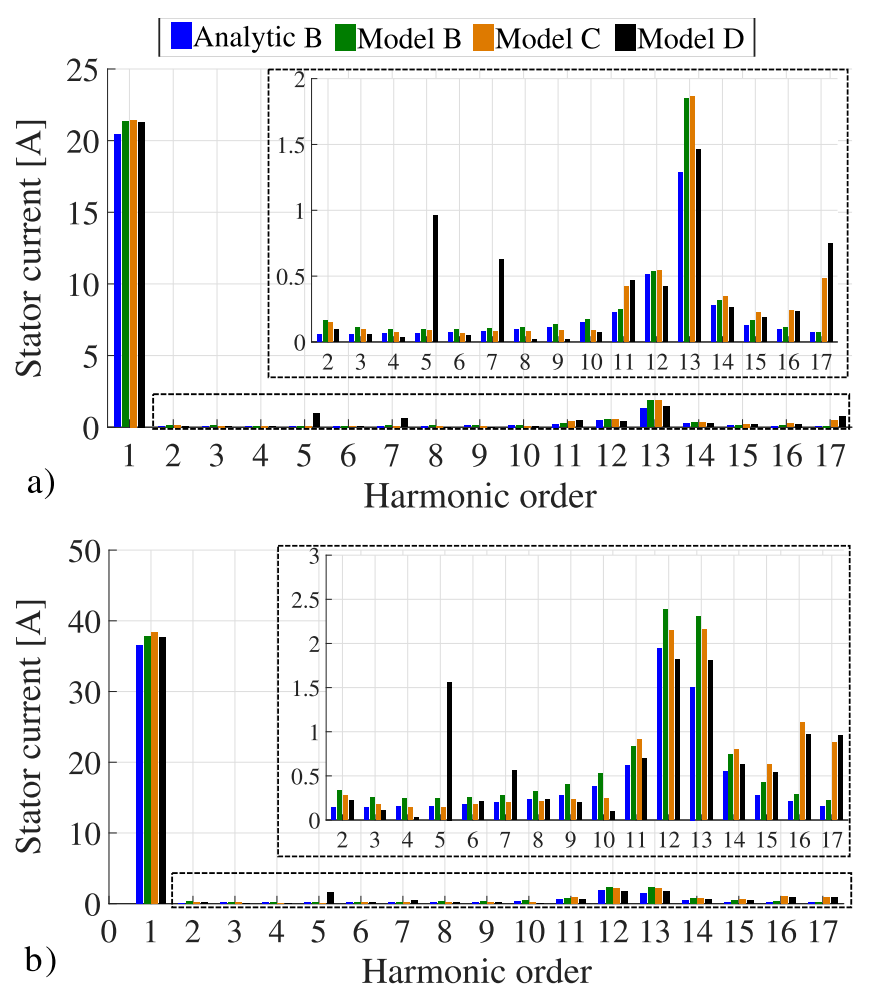

Fig. 11: Stator current spectra at a) $s=0.02$ and b) $s=0.04$.

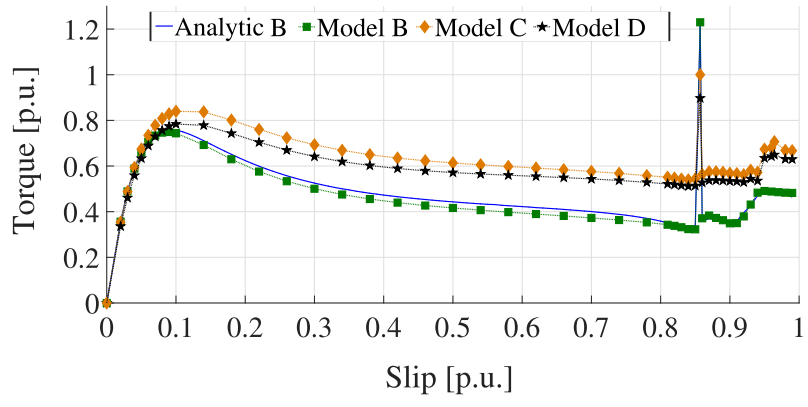

Fig. 12: Stator current harmonic spectrum at $s=0.02$.

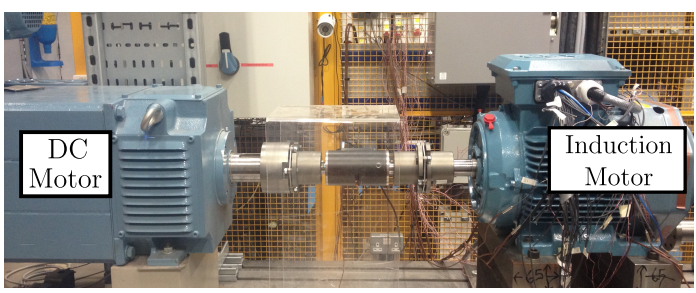

Fig. 13: The experimental platform

\section{CONCLUSION}

An analytical-numerical resolution of the governing equations which describes the behaviour of SCIMs is proposed. Description of the model improvements introduced to account for the slotting effects, MMF harmonics and skewing are presented. The model reduction proposed by exploiting the symmetries and anti-symmetries of the rotor cage permits to reduce drastically the computational burden and avoid in first approximation the computation of end rings leakage inductance when compared against FEA. The model is validated through a series of FE models featuring increased amount of more realistic details and experimental results for a SCIM featuring closed slot and skewed rotor cage structure.

The proposed model proves to be an effective approach which is demonstrated to sit between the FE and EC modelling technique for SCIMs in terms of accuracy and computational effort, thus permitting to predict the time dependency of stator and eventually rotor currents as well as the torque profile. The phenomena due to the MMF harmonics interaction are successfully predicted. At high slip values the synchronous and asynchronous torque occurred and the effects of a skewed rotor in mitigating them, can be studied with reduced computational time with respect to FE analysis. The flexibility guaranteed by the modelling approach allows to investigate different motor configurations. The model proposed is finally a good candidate to be employed for implementing control strategies that require accurate models featuring multi-space harmonic orders.

\section{REFERENCES}

[1] A. T. de Almeida, F. J. T. E. Ferreira, and G. Baoming, "Beyond induction motors-technology trends to move up efficiency," IEEE Transactions on Industry Applications, vol. 50, no. 3, pp. 2103-2114, May 2014.

[2] S. A. Odhano, R. Bojoi, A. Boglietti, G. Roşu, and G. Griva, "Maximum efficiency per torque direct flux vector control of induction motor drives," IEEE Transactions on Industry Applications, vol. 51, no. 6, pp. 4415-4424, Nov 2015. 

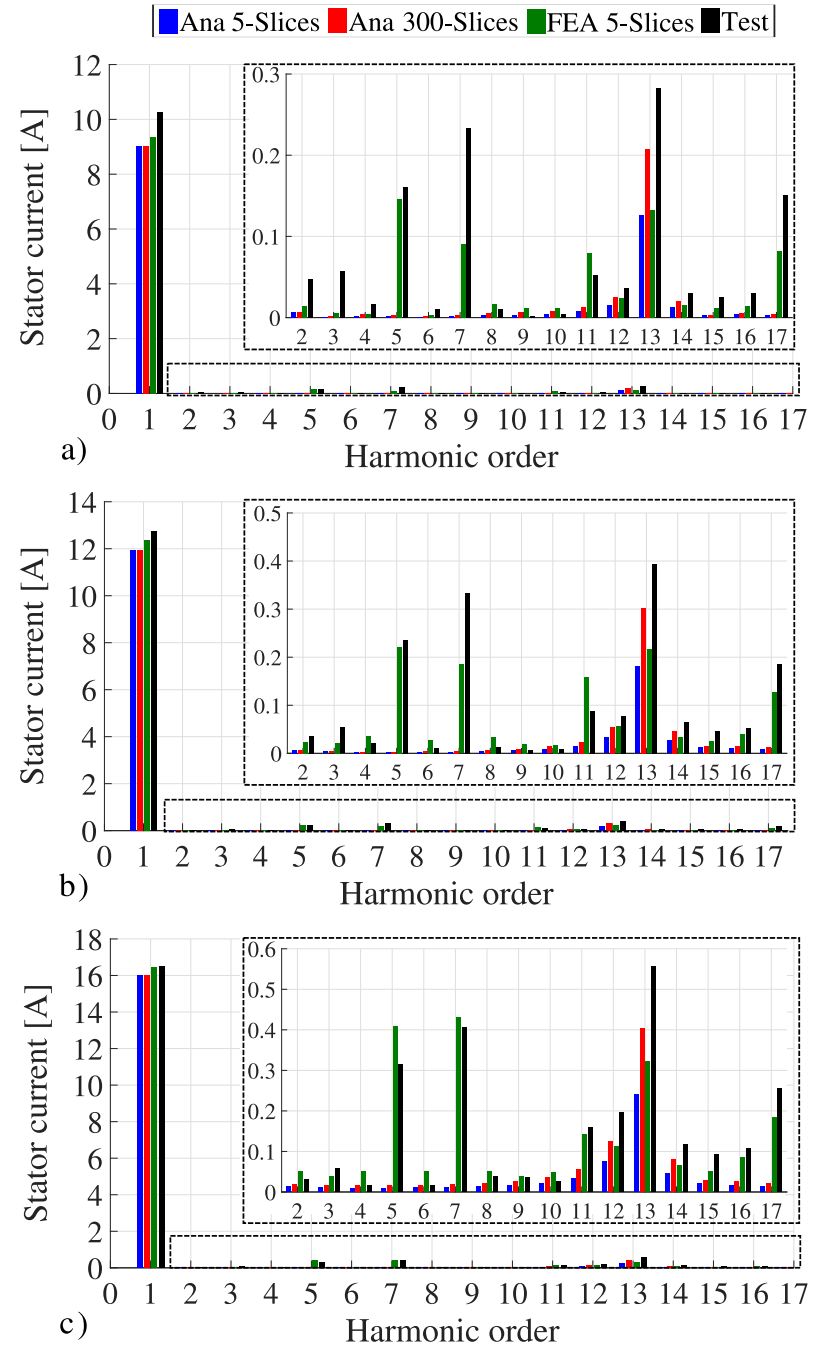

Fig. 14: Stator current spectra: a) $50 \%$, b) $70 \%$ and c) $100 \%$ of the rated load.

[3] A. T. De Almeida, F. J. T. E. Ferreira, and A. Q. Duarte, "Technical and economical considerations on super high-efficiency three-phase motors," IEEE Transactions on Industry Applications, vol. 50, no. 2, pp. 12741285, March 2014.

[4] A. Marfoli, G. Sala, L. Papini, P. Bolognesi, and C. Gerada, "Torque ripple investigation in squirrel cage induction machines," in 2019 IEEE International Electric Machines Drives Conference (IEMDC), May 2019, pp. 140-146.

[5] L. Alberti, N. Bianchi, and S. Bolognani, "A very rapid prediction of im performance combining analytical and finite-element analysis," IEEE Transactions on Industry Applications, vol. 44, no. 5, pp. 1505-1512, Sept 2008.

[6] A. Marfoli, L. Papini, P. Bolognesi, D. Genovese, and C. Gerada, "Analysis of induction machine: Comparison of modelling techniques," in 2017 IEEE International Electric Machines and Drives Conference (IEMDC), May 2017, pp. 1-7.

[7] D. Genovese, P. Bolognesi, M. D. Martin, and F. Luise, "A contextual parameter identification method for the equivalent circuit of induction machine," in 2016 XXII International Conference on Electrical Machines (ICEM), Sept 2016, pp. 25-31.

[8] M. De Martin, M. Bailoni, A. Tessarolo, M. Bortolozzi, D. Giulivo, F. Agnolet, and R. Santarossa, "Investigation into induction motor equivalent circuit parameter dependency on current and frequency variations," in 2014 International Conference on Electrical Machines (ICEM), Sep. 2014, pp. 196-202.

[9] I. Boldea and S. A. Nasar, The induction machines design handbook. CRC press, 2018.

[10] A. R. Munoz and T. A. Lipo, "Complex vector model of the squirrel-cage induction machine including instantaneous rotor bar currents," IEEE Transactions on Industry Applications, vol. 35, no. 6, pp. 1332-1340,
Nov 1999.

[11] H. A. Toliyat and T. A. Lipo, "Transient analysis of cage induction machines under stator, rotor bar and end ring faults," IEEE Transactions on Energy Conversion, vol. 10, no. 2, pp. 241-247, Jun 1995.

[12] G. Joksimovic, "Parameterized dynamic model of cage induction machine," in 2016 XXII International Conference on Electrical Machines (ICEM), Sept 2016, pp. 513-518.

[13] S. Nandi, "Modeling of induction machines including stator and rotor slot effects," IEEE Transactions on Industry Applications, vol. 40, no. 4, pp. 1058-1065, July 2004.

[14] G. Joksimović, J. Riger, T. Wolbank, N. Perić, M. Vašak, G. Stojčić, and V. Lešić, "Dynamic induction machine model accounting for stator and rotor slotting," in 2012 XXth International Conference on Electrical Machines, Sept 2012, pp. 207-212.

[15] B. Heller and V.Hamata, Harmonic field effects in Induction Machine, N. Y. Elsevier, Ed., 1977.

[16] P. Bolognesi, "Generalized circuital modeling of electromagnetic devices," Proc. of ICEM 2004 Conf., Cracow, Sep. 2004., 2004.

[17] A. Marfoli, P. Bolognesi, L. Papini, and C. Gerada, "Mid-complexity circuital model of induction motor with rotor cage: A numerical resolution," in 2018 XIII International Conference on Electrical Machines (ICEM), Sep. 2018, pp. 277-283.

[18] P. Bolognesi, "A mid-complexity analysis of long-drum-type electric machines suitable for circuital modeling," in 2008 18th International Conference on Electrical Machines, Sept 2008, pp. 1-5.

[19] R. De Weerdt and R. Belmans, "Squirrel cage induction motor end effects using 2d and 3d finite elements," in 1995 Seventh International Conference on Electrical Machines and Drives (Conf. Publ. No. 412), Sep. 1995, pp. 62-66.

[20] S. Nuzzo, P. Bolognesi, C. Gerada, and M. Galea, "Simplified damper cage circuital model and fast analytical-numerical approach for the analysis of synchronous generators," IEEE Transactions on Industrial Electronics, pp. 1-1, 2018.

[21] T. S. Birch and O. I. Butler, "Permeance of closed-slot bridges and its effect on induction-motor-current computation," Proceedings of the Institution of Electrical Engineers, vol. 118, no. 1, pp. 169-172, January 1971. 